\section{Welcome to the Swedish theme issue: putting exercise into sports medicine}

\author{
Richard Frobell, ${ }^{1}$ Mats Börjesson ${ }^{2}$
}

This theme issue celebrates the collaboration between BJSM and the Swedish Society of Exercise and Sports Medicine (SFAIM). From this issue onwards, SFAIM members will gain full access to BJSM through a link on our website (http:// www.svenskidrottsmedicin.se); SFAIM will also reach out to a wide audience using the BJSM blog and podcasts. As the chairman of SFAIM, I am proud to be guest editing this issue, together with Richard Frobell, where we present some of the ongoing work of Swedish Exercise and Sports Medicine.

\section{SFAIM: A PROUD TRADITION}

SFAIM was formed in 1952; it was affiliated with the Swedish Society of Medicine and the Féderation Internationale de Médecine du Sport (FIMS) within its inaugural year. From the beginning, physiology and traumatology featured prominently in the Society, illustrated by the topics from the first scientific meetings in 1952: 'Ligament Injury of the Knee in Sports', 'Tennis Elbow', 'Eye Injuries in Ice hockey' and 'Knockout in Boxing'. In 1953, 'The Athlete's Heart' was discussed, followed by 'Testing methods for Physical Performance' and 'Achilles Tendon Ruptures'. Interestingly, these topics are still highly relevant and appear in 2013 Sports Medicine conference programmes!

Has nothing changed, then? Indeed, it has. Our society as well as the overall health panorama have changed markedly in the past 60 years. Today, a top health challenge is the increasing frequency of lifestyle related diseases, and their consequences. Medical care, including new and more effective medications, the reduction of some important risk factors (such as smoking), improved surgical techniques and postoperative rehabilitation has markedly decreased the risk of cardiovascular death in the Western world. However, in most other areas of the world,

\footnotetext{
${ }^{1}$ Department of Orthopedics, Clinical Sciences Lund, Lund University, Lund, Sweden; ${ }^{2}$ Swedish School of Sport and Health Sciences and Karolinska University Hospital, Stockholm, Sweden
}

Correspondence to Dr Richard Frobell, Department of Orthopedics, Clinical Sciences Lund, Lund University, Lund 22185, Sweden; Richard.frobell@med.lu.se cardiovascular deaths are still on the rise. Unhealthy lifestyles, including physical inactivity, are a major driving force of this trend, and our community faces a major challenge to reverse it. The Sports Medicine community is becoming increasingly aware of this assignment, but how could we help to counteract the problem?

\section{EMBRACING A COMPLEMENTARY NEW AGENDA: EXERCISE ON PRESCRIPTION}

We believe that the (historical) emphasis on elite sports needs to be complemented by an increasing awareness of the importance of 'sports for health' initiatives. This awareness has been reflected in recent name changes of many Sports Medicine societies, such as the 'The Swedish Society of Exercise and Sports Medicine' and 'The British Association of Sport and Exercise Medicine'. As early as the 1940s, there was a discussion in Sweden regarding the health benefits and risks for untrained individuals in participating in 'Riksmarschen', a national public running race. Interestingly, this debate continues with respect to marathon running, crosscountry ski races, etc. Sports cardiology for example, which traditionally focused on sudden cardiac death in younger athletes, now plays an important preventive role by formulating screening and eligibility criteria also for older athletes. The goal is to enable safe exercise participation for everyone.

\section{FYSS AND THE SWEDISH PHYSICAL ACTIVITY BOOK}

To win the war against lifestyle-related disease progression, we believe that continuing the work on 'exercise prescription for health' is of utmost importance for our society and Sports Medicine at large. In the late 1990s, SFAIM brought together the Swedish national expertise to produce a handbook, FYSS (http:// www.fyss.se), aimed at collecting the scientific evidence for the health benefits of physical activity for 33 different conditions from A (anxiety) to $\mathrm{Z}$ (well, $\mathrm{S}$ for stroke). FYSS provides information on which type of physical activity (as well as duration, frequency and intensity) has the best proven effect, what is the mechanism of action and what are the indications and contraindications for prescribing physical activity. The book outlines interactions of physical activity with medical treatment to aid in formulating a 'prescription of physical activity' for a wide spectrum of conditions. FYSS is gaining adoption internationally and has been translated into Norwegian, English and Vietnamese. The greatest challenge for exercise prescription may lie ahead; however, to convince and motivate our colleagues to incorporate exercise and physical activity as a regular treatment modality in modern health care is one of SFAIMs most important goals. Read more about FYSS in the accompanying Editorial (see page 937).

\section{YOU CAN JOIN SFAIM AND OUR 2014 CONFERENCE IS IN MAY!}

SFAIM's mission is to promote physical activity as an important factor for improved health in the general population and to promote safe sports participation. You can become a member of SFAIM if you have a dedicated interest in sports medicine and physical activity. The regional chapters of SFAIM (see the website) organise regular local meetings around specific topics. Our national annual scientific meeting takes place each spring (2014 meeting in Stockholm 8-10 May, http:// www.svenskidrottsmedicin.se). As well as facilitating multidisciplinary discussions, SFAIM have designated sections for physiotherapists, naprapaths (the Swedish health profession), chiropractors and medical doctors. We have a dedicated 'sports traumatology' section as well as a section dedicated to the continued updating and implementation of FYSS named YFA (Yrkesföreningar för fysisk aktivitet/Professional Associations for Physical Activity).

\section{IN THIS SWEDISH ISSUE OF BJSM}

We are proud to present this issue of $B J S M$ which exemplifies the current diversity and high activity of SFAIM:

Public health and exercise science is presented in the Editorial by Sundberg/ Borjesson (see page 937) where FYSS is outlined and explored, and in the report from Nyberg et al in which a new maximal step-up test is shown to be a simple, robust and relevant way to assess leg strength and function in primary care patients (see page 992). The authors suggest that maximal step-up height is a useful health indicator for general practice.

Sports traumatology is nicely covered by the paper on injury patterns in Swedish track-and-field (see page 941), as well as the trilogy of papers (all by Ryynänen et al) from the very highest level of football, the 
FIFA World Cup. Here, the association between injury incidence and scores (see page 960), potential game disrupting events (see page 970), and foul play (see page 986), are presented. Increased knowledge in these areas is important to prevent future injuries.

Research on rehabilitation and physical function is represented with three papers: Askling et al (see page 953), compare two rehabilitation protocols in a randomised controlled trial of hamstring injuries in European football; Hägglund et al (see page 974), compare the compliance rate of a neuromuscular preventive training regime, with subsequent knee-injury rates in football; Eriksson et al (see page 980), present and compare functional performance test results at the end of the exercise period between anterior cruciate ligament (ACL) injured individuals treated with and without ACL reconstruction.

The effects of doping are further presented in the paper on long-term mental effects of steroid use by Lindqvist et al (see page 965), where former doping users and power athletes from Sweden were investigated after 30 years.

To summarise, SFAIM is proud of its long-standing traditions in the delivery of Sports and Exercise Medicine care of athletes as well as in quality scientific activity. We are pleased to have BJSM support us in taking those goals forwardclinical and public health excellence through research, education and implementation of evidence-based programmes.

Competing interests None.

Provenance and peer review Not commissioned; internally peer reviewed.

To cite Frobell $\mathrm{R}$, Börjesson M. Br J Sports Med 2013;47:935-936.

Accepted 7 August 2013

Br J Sports Med 2013;47:935-936. doi:10.1136/bjsports-2013-092971 